The Journal of Rheumatology 2022;49:2

doi:10.3899/jrheum.210968

First Release September 152021

\section{Dr. Berti et al reply}

We read with interest the comment of Dr. Kawada ${ }^{1}$ on our article that showed an inverse association between current smoking status and primary (p-) Sjögren syndrome (SS), and no association between obesity and $\mathrm{pSS}{ }^{2}$

The relationship between smoking and the development of systemic autoimmune diseases has been explored by numerous authors. For some diseases such as rheumatoid arthritis (RA), the link appears well established, whereas for others the relationship is more complex. As Dr. Kawada comments, 2 more recently published articles, using even larger numbers of patients than our population-based cohort on the relationship between smoking and $\mathrm{pSS}^{3}$ and on SS among patients with RA, ${ }^{4}$ confirmed the main finding of our study (i.e., the negative correlation between current smoking and SS); they also added other variables of potential interest such as the temporal smoking patterns preceding $\mathrm{pSS}$ diagnosis ${ }^{3}$ and the association of age and female sex with SS in RA. ${ }^{4}$ The relationship between smoking and disease occurrence may hold in both primary and secondary SS. ${ }^{4}$ It is our view that these epidemiological associations may provide some insight into the pathogenesis of disease and require further study at the genomic and proteomic level.

Alvise Berti ${ }^{1}(\mathbb{D}, \mathrm{MD}$

Divi Cornec ${ }^{2}$ (D) $\mathrm{MD}, \mathrm{PhD}$

Cynthia S. Crowson ${ }^{3}$ (D) PhD

Eric L. Matteson ${ }^{4}(\mathbb{D}, \mathrm{MD}, \mathrm{MPH}$

${ }^{1}$ University of Trento and Santa Chiara Hospital, Trento, Italy;

${ }^{2}$ Rheumatology Department, Brest Teaching Hospital, Brest,

France;

${ }^{3}$ Division of Clinical Trials and Biostatistics, Department of

Quantitative Health Sciences, and Division of Rheumatology,

Mayo Clinic College of Medicine and Science, Rochester,

Minnesota, USA;

${ }^{4}$ Division of Rheumatology, and Division of Epidemiology,

Department of Health Sciences Research, Mayo Clinic College

of Medicine and Science, Rochester, Minnesota, USA.
This work was made possible using the resources of the Rochester Epidemiology Project, which is supported by the National Institute on Aging of the National Institutes of Health (NIH) under Award Number R01AG034676, and Clinical and Translational Science Awards Grant Number UL1 TR000135 from the National Center for Advancing Translational Sciences, a component of the NIH. The content is solely the responsibility of the authors and does not necessarily represent the official views of the NIH.

The authors declare no conflicts of interest relevant to this article.

Address correspondence to Dr. E.L Matteson, Division of Rheumatology, and Division of Epidemiology, Department of Health Sciences Research, Mayo Clinic College of Medicine and Science, 200 1st St. SW, Rochester, MN 55902, USA. Email: matteson.eric@mayo.edu.

\section{REFERENCES}

1. Kawada T. Smoking, obesity, and risk of primary Sjögren syndrome. J Rheumatol 2022; 49:234.

2. Servioli L, Maciel G, Nannini C, et al. Association of smoking and obesity on the risk of developing primary Sjögren syndrome: a population-based cohort study. J Rheumatol 2019;46:727-30.

3. Mofors J, Björk A, Richardsdotter Andersson E, et al. Cigarette smoking patterns preceding primary Sjögren's syndrome. RMD Open 2020;6:e001402.

4. McCoy SS, Greenlee RT, VanWormer JJ, Schletzbaum M, Bartels CM. Smoking associated with reduced odds of Sjögren's syndrome among rheumatoid arthritis patients. Scand J Rheumatol 2021 Jun 25 (Epub ahead of print). 